\title{
Non-human primate research of basal ganglia and movement disorders: advances and challenges
}

\author{
Yoland Smith ${ }^{1} \cdot$ Adriana Galvan ${ }^{1}$
}

Published online: 8 February 2018

C) Springer-Verlag GmbH Austria, part of Springer Nature 2018

Research in nonhuman primates (NHPs) has played a critical role in advancing knowledge of the anatomy and physiology of the basal ganglia over the past 50 years. Furthermore, the 1-methyl-4-phenyl-1,2,3,6-tetrahydropyridine (MPTP)treated NHP model of Parkinson's disease (PD) has been instrumental in the rejuvenation of surgical therapies for Parkinson's disease and related movement disorders. Because the basal ganglia nuclei are divided and functionally compartmentalized in the same fashion in monkeys and humans, NHPs represent the most human-like related species to study basal ganglia organization in normal and diseased states. Another striking evolutionary feature that makes NHPs, in particular Old World monkeys (macaques, baboons, etc...), an ideal model to study basal ganglia function and dysfunction, is the significant expansion and complexity of the cerebral cortex in these primates compared with rodents or other non-primate species. In particular, the extensive development of high-order associative cortical regions provide monkeys human-like cognitive capabilities that cannot be assessed in any other animal species. The fact that most regions of the cerebral cortex are closely connected with the basal ganglia, and that dysfunction of these cortical areas, and their relationships with the basal ganglia, contribute to the pathophysiology of neurological and psychiatric disorders further highlight the importance of NHP research to further understand the neurobiology of these disorders and the development of appropriate therapeutic approaches.

Despite their obvious value, NHPs account for less than $1 \%$ of all laboratory animals used in biomedical research. The increased costs and regulations, combined with pressure from groups opposed to NHP experimentation, have led to a dramatic reduction in the use of monkeys in neuroscience

Yoland Smith

ysmit01@emory.edu

1 Department of Neurology, Yerkes National Primate Research Center, Emory University, 954, Gatewood Rd NE, Atlanta, GA 30329, USA research. This is a non-sustainable path forward because our knowledge of the human brain will rely almost entirely on data from rodent experiments which, in many cases, are gathered from functional brain networks that are much less complex and extensive than in primates. A significant knowledge gap in our understanding of the human brain, and in the development of adequate therapies for complex human brain diseases may result from this continuous lack of NHP experimentation. There is an urgent need for funding agencies to carefully examine the current path of neuroscience research support and put in place mechanisms to encourage and prioritize high-standard NHP research that addresses fundamental issues relevant to the human brain.

In this Special Issue, we have invited various NHP research teams to write reviews on key topics relevant to the functional anatomy and pathophysiology of basal ganglia circuits in normal and diseased states. Many reviews discuss the relevance and impact of NHP models to study motor and non-motor functions associated with PD, and to develop new therapeutic approaches for this disorder. These are followed by discussions about the role of basal ganglia circuits in learning and memory, and the potential impact of dysregulation of these networks in cognitive deficits associated with PD and other neurodegenerative disorders. Finally, the Special Issue concludes with manuscripts that highlight the challenges in translating genetic-based approaches, commonly used in mice to manipulate and repair specific brain networks, to studies of the NHP brain.

In the first manuscript, Petryszyn et al. (2017) review evidence for an important species difference between rodents and primates in the chemical phenotype and prevalence of a specific subset of large calretinin-positive interneurons that co-express acetylcholine in the monkey striatum. Because these neurons are non-existent in rodents, and undergo significant alterations in diseased states, the use of NHPs to understand their role in normal basal ganglia functions and in the pathophysiology of basal ganglia disorders is essential. This manuscript is followed by a series of reviews on the use 
of the MPTP-treated NHP model of PD to study the pathogenesis, and test new therapies for PD and L-DOPA-induced dyskinesias (Morissette and Di Paolo 2017; Blesa et al. 2017; Masilamoni and Smith 2017; Vermilyea and Emborg 2017). In addition, Masilamoni and Smith (2017) highlight the fact that the chronic intoxication of monkeys with MPTP results in an animal model that displays brain pathology that extends far beyond the midbrain dopaminergic cell groups to include other brainstem monoaminergic neurons and intralaminar thalamic cells, suggesting that chronically MPTPtreated monkeys can potentially be used as an animal model to study the pathophysiology and therapeutics of non-motor symptoms of PD. Vermilyea and Emborg (2017) discuss the value of the MPTP-treated monkey model for the development and testing of novel cell-based therapies that could potentially be used in PD patients. To follow-up this series of discussions focused on the MPTP-treated monkey model, Marmion and Kordower (2017) bring our attention to a new generation of NHP models of PD based on the overexpression of alpha-synuclein in midbrain dopaminergic neurons and their striatal projections. Another promising avenue for the development of NHP model of neurodegenerative diseases is the use of transgenic approaches. In their manuscript, Snyder and Chan (2017) presents recent evidence for the development of a transgenic rhesus monkey model for Huntington's disease. Efforts from other groups are being devoted towards the development of transgenic monkeys for other brain disorders, including PD. Despite significant challenges, this approach may lead to a new generation of NHP models that could have an impact on the development and testing of neuroprotective strategies for neurodegenerative diseases.

One of the major contributions NHP models have made to the field of basal ganglia and PD research is to deepen our understanding of the pathophysiology of basal ganglia circuits and the subsequent development of surgical therapies for PD and other movement disorders (in particular dystonia). In their review, Wichmann et al. (2017) discuss the literature that set the foundation for the development and refinement of deep brain stimulation (DBS) for movement disorder therapy, and highlight the impact of this therapeutic approach for patients affected with advanced PD and dystonia. This manuscript is followed by three reviews that cover key pathophysiological features that contribute to basal ganglia network dysfunction in PD. Villalba and Smith (2017) review the literature about the plastic changes in the morphology of striatal projection neurons and their synaptic glutamatergic afferents. The possible role of these structural alterations in the abnormal transmission and plasticity of the glutamatergic corticostriatal and thalamostriatal systems is highlighted. Beck et al. (2017) discuss recent evidence from their laboratory showing that the physiological activity of striatal projection neurons is altered in both MPTP-treated parkinsonian monkeys and PD patients, while Merchant and Bartolo (2017) discuss the potential importance of striatal beta oscillations in guiding rhythmic movements, and highlight the significance of changes in these oscillations in PD. It has become clear over the past decades that PD is far more than a mere motor disorder induced by selective loss of midbrain dopaminergic neurons and the degeneration of the nigrostriatal system. One brain region that has generated significant interest in recent years because of its potential role in gait and sleep control is the pedunculopontine tegmental region (PPN). Karachi and Francois (2017) describe the key anatomical, physiological and pathophysiological features of the PPN and the potential involvement of its degeneration in gait problems often seen in PD patients. In addition to the cardinal motor signs, PD patients suffer from a wide range of psychiatric, autonomic and cognitive dysfunctions that likely result from degeneration of non-dopaminergic systems. In their review, Sgambato-Faure and Tremblay (2017) highlight the potential importance of degeneration of the ascending serotonergic projections to the ventral striatopallidal complex and prefrontal cortical regions in the development of neuropsychiatric deficits in PD.

In primates, prefrontal, parietal and temporal associative cortical regions are major sources of inputs to the caudate nucleus, and a significant territory of each basal ganglia nuclei is devoted to cognitive processing. Thus, not surprisingly, brain diseases that affect the basal ganglia are often characterized by various degrees of cognitive impairments in learning and memory. Yamanaka et al. (2017) discuss the importance of projections from the centromedian and parafascicular thalamic nuclei to striatal cholinergic interneurons in associative learning of environmental events. They also highlight the fact that the degeneration of these thalamic nuclei in PD and other neurodegenerative diseases may contribute to cognitive impairments in these disorders. Another key contribution about the role of basal ganglia in cognition comes from Hikosaka et al. (2017) who provide a thorough review of their work and others showing that specific basal ganglia circuits can encode values of many behaviors and retain the value signals of these behaviors for a long time. They discuss the anatomy and physiology of these circuits, and the critical role played by dopamine in regulating their contribution to decision-making. In addition to the neuropsychiatric disorders discussed above that may result from basal ganglia network dysfunction and degeneration, there is strong evidence that the basal ganglia may also be involved in the control of focal and generalized seizures. Vuong and Devergnas (2017) provide a comprehensive account of the literature about the role of the basal ganglia in absence, temporal lobe, and neocortical seizures, and discuss the relevance of NHP models to assess the mechanisms of seizure propagation and the potential therapeutic benefit of DBS in treating various forms of seizure disorders. 
This Special Issue concludes with three reviews that highlight cutting edge viral vector strategies that must be successfully translated from their use in mice to nonhuman primates if one hopes to advance knowledge of the anatomical and functional organization of basal ganglia networks, and develop new gene therapy-based approaches to fix these systems in diseased states. Galvan et al. (2017) provide an update of the current use of optogenetic and chemogenetic approaches to interrogate specific neuronal subtypes and their connections in monkeys. This review highlights the significant challenges these genetic-based approaches face when applied to the primate brain. Alikaya et al. (2017) address another major technical challenge that must be overcome to allow manipulation of specific cell types in the monkey brain. In this manuscript, the authors review some of their recent data gathered through the use of a dual viral vector strategy to selectively regulate midbrain dopaminergic neurons and study their role in reward and value coding. Efforts must be devoted to assess the reliability of this powerful approach to target other specific neuronal subtypes in the monkey brain. In the last manuscript, Pignataro et al. (2017) highlight the importance of NHP models of brain diseases for the development of new gene-therapy approaches that could treat basal ganglia-related disorders in humans. The large size of the brain and the different genetic make-up of monkeys vs rodents compared with humans, are fundamental issues that make the use of NHPs absolutely essential to translate these genetic-based approaches to human brain therapeutics.

In conclusion, NHPs have been instrumental in advancing our understanding of basal ganglia function in normal and diseased states for the past 50 years. Continued progress in the use of NHPs to help advance our understanding of functional brain connectivity and disruptions of these networks in neurological diseases rely on the successful translation of genetic-based technical and therapeutic approaches widely used in mice, to the NHP brain. As highlighted in some of the reviews presented in this Special Issue, achieving this ultimate goal faces significant technical challenges. These, combined with other problems of increased costs, regulations and pressure from animal rights group, represent significant impediments to advances in our understanding of the human brain and to the development of innovative therapies for devastating human brain disorders, through the use of NHPs. Although rodent research is of tremendous importance and has had a major impact in various fields of neuroscience, the need for NHPs is absolutely essential to bridge the knowledge gap in brain anatomy and function between humans and non-human species. Similarly, efforts in developing new monkey models of brain diseases are critical to the translation of future therapies to human diseased conditions. To achieve these goals, various initiatives will have to be put in place by funding agencies to incentivize the development of innovative primate-specific toolboxes that can be reliably applied to the monkey brain to study function and dysfunction of neuronal networks in normal and diseased states.

\section{References}

Alikaya A, Rack-Wildner M, Stauffer WR (2017) Reward and value coding by dopamine neurons in non-human primates. J Neural Transm (Vienna). https://doi.org/10.1007/s00702-017-1793-9

Beck G, Singh A, Papa SM (2017) Dysregulation of striatal projection neurons in Parkinson's disease. J Neural Transm (Vienna). https ://doi.org/10.1007/s00702-017-1744-5

Blesa J, Trigo-Damas I, Del Rey NL, Obeso JA (2017) The use of nonhuman primate models to understand processes in Parkinson's disease. J Neural Transm (Vienna). https://doi.org/10.1007/s0070 2-017-1715-x

Galvan A, Caiola MJ, Albaugh DL (2017) Advances in optogenetic and chemogenetic methods to study brain circuits in non-human primates. J Neural Transm (Vienna). https://doi.org/10.1007/s0070 2-017-1697-8

Hikosaka O, Ghazizadeh A, Griggs W, Amita H (2017) Parallel basal ganglia circuits for decision making. J Neural Transm (Vienna). https://doi.org/10.1007/s00702-017-1691-1

Karachi C, Francois C (2017) Role of the pedunculopontine nucleus in controlling gait and sleep in normal and parkinsonian monkeys. J Neural Transm (Vienna). https://doi.org/10.1007/s0070 2-017-1678-y

Marmion DJ, Kordower JH (2017) $\alpha$-Synuclein nonhuman primate models of Parkinson's disease. J Neural Transm (Vienna). https ://doi.org/10.1007/s00702-017-1720-0

Masilamoni GJ, Smith Y (2017) Chronic MPTP administration regimen in monkeys: a model of dopaminergic and non-dopaminergic cell loss in Parkinson's disease. J Neural Transm (Vienna). https ://doi.org/10.1007/s00702-017-1774-z

Merchant H, Bartolo R (2017) Primate beta oscillations and rhythmic behaviors. J Neural Transm (Vienna). https://doi.org/10.1007/ s00702-017-1716-9

Morissette M, Di Paolo T (2017) Non-human primate models of PD to test novel therapies. J Neural Transm (Vienna). https://doi. org/10.1007/s00702-017-1722-y

Petryszyn S, Parent A, Parent M (2017) The calretinin interneurons of the striatum: comparisons between rodents and primates under normal and pathological conditions. J Neural Transm (Vienna). https://doi.org/10.1007/s00702-017-1687-x

Pignataro D, Sucunza D, Rico AJ, Dopeso-Reyes IG, Roda E, Rodriguez-Perez AI, Labandeira-Garcia JL, Broccoli V, Kato S, Kobayashi K, Lanciego JL (2017) Gene therapy approaches in the non-human primate model of Parkinson's disease. J Neural Transm (Vienna). https://doi.org/10.1007/s00702-017-1681-3

Sgambato-Faure V, Tremblay L (2017) Dopamine and serotonin modulation of motor and non-motor functions of the non-human primate striato-pallidal circuits in normal and pathological states. J Neural Transm (Vienna). https://doi.org/10.1007/s0070 2-017-1693-z

Snyder BR, Chan AWS (2017) Progress in developing transgenic monkey model for Huntington's disease. J Neural Transm (Vienna). https://doi.org/10.1007/s00702-017-1803-y

Vermilyea SC, Emborg ME (2017) The role of nonhuman primate models in the development of cell-based therapies for Parkinson's disease. J Neural Transm (Vienna). https://doi.org/10.1007/s0070 2-017-1708-9 
Villalba RM, Smith Y (2017) Loss and remodeling of striatal dendritic spines in Parkinson's disease: from homeostasis to maladaptive plasticity? J Neural Transm (Vienna). https://doi.org/10.1007/ s00702-017-1735-6

Vuong J, Devergnas A (2017) The role of the basal ganglia in the control of seizure. J Neural Transm (Vienna). https://doi.org/10.1007/ s00702-017-1768-X

Wichmann T, Bergman H, DeLong MR (2017) Basal ganglia, movement disorders and deep brain stimulation: advances made through non-human primate research. J Neural Transm (Vienna). https://doi.org/10.1007/s00702-017-1736-5

Yamanaka K, Hori Y, Minamimoto T, Yamada H, Matsumoto N, Enomoto K, Aosaki T, Graybiel AM, Kimura M (2017) Roles of centromedian parafascicular nuclei of thalamus and cholinergic interneurons in the dorsal striatum in associative learning of environmental events. J Neural Transm (Vienna). https://doi. org/10.1007/s00702-017-1713-Z 